\title{
Recent Advances in
} PSORIASIS

\author{
The Role of the \\ Immune System
}




\section{Barbara S Baker}

Imperial College School of Medicine of St Mary's, London

Recent Advances in

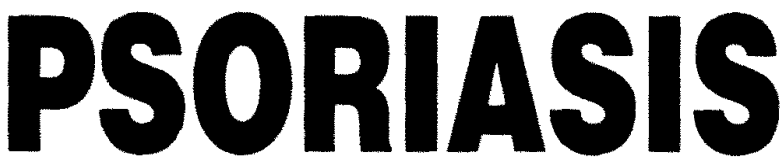

The Role of the
Immune System

Imperial College Press 
Published by

Imperial College Press

57 Shelton Street

Covent Garden

London WC2H 9HE

\section{Distributed by}

World Scientific Publishing Co. Pte. Ltd.

P O Box 128, Farrer Road, Singapore 912805

USA office: Suite 1B, 1060 Main Street, River Edge, NJ 07661

UK office: 57 Shelton Street, Covent Garden, London WC2H 9HE

\section{British Library Cataloguing-in-Publication Data}

A catalogue record for this book is available from the British Library.

\section{RECENT ADVANCES IN PSORIASIS: THE ROLE OF THE IMMUNE SYSTEM}

Copyright $\odot 2000$ by Imperial College Press

All rights reserved. This book, or parts thereof, may not be reproduced in any form or by any means, electronic or mechanical, including photocopying, recording or any information storage and retrieval system now known or to be invented, without written permission from the Publisher.

For photocopying of material in this volume, please pay a copying fee through the Copyright Clearance Center, Inc., 222 Rosewood Drive, Danvers, MA 01923, USA. In this case permission to photocopy is not required from the publisher.

ISBN $1-86094-120-6$

This book is printed on acid and chlorine free paper.

Printed in Singapore by Fulsland Offset Printing 


\section{Preface}

Psoriasis is considered as a disease of epidermal hyperproliferation induced by $T$ lymphocytes. The purpose of this book is to bring together the various pieces of evidence that have contributed to our current understanding of the role of the immune system in psoriasis. The latest approaches to investigating the immunopathogenesis of this disease, and a review of previous findings, are presented to give an overall picture of the current knowledge in this field. Novel therapies to treat this disease are described, some of which are already being tested on patients. Unlike current treatments, these new approaches have the potential to switch off the disease process on a long-term basis. In addition, a model is proposed for the immunopathogenesis of psoriasis which hopefully will serve as a basis for further research.

The findings presented here illustrate the considerable progress that has been been achieved in recent years in our understanding of the immunopathogenic mechanisms involved in this common skin disease. 
vi Recent Advances in Psoriasis: The Role of the Immune System

I would like to thank Professor Lionel Fry with whom I have collaborated on this work over a period of almost 20 years, and who continues to give me support and encouragement.

Barbara S. Baker Imperial College School of Medicine at St. Mary's London 


\section{Contents}

Preface v

Chapter 1 Aetiology, Clinical and Histological Features of Psoriasis

1.1 Clinical Features

1.2 Genetics

1.2.1 Epidemiology

1.2.2 Mode of Inheritance 3

1.2.3 Genomic Imprinting 4

1.2.4 HLA Associations 4

1.2.5 Gene Linkage 6

1.2.6 Candidate Genes 8

1.3 Environmental Factors 9

1.4 Histological Features $\quad 10$

Chapter 2 Skin Immune System in Psoriasis $\quad 14$

2.1 Skin Immune System (SIS) 14

2.2 T Cell Subpopulations: Normal Skin 16

2.3 T Cell Subpopulations: Psoriatic Skin $\quad 17$

2.4 Antigen-Presenting Cells: Normal Skin 21

2.4.1 Epidermal Dendritic Cells 21

2.4.2 Dermal Dendritic Cells (DDC) 23

2.4.3 Indeterminate Cells 23

2.4.4 Macrophages 24 
viii Recent Advances in Psoriasis: The Role of the Immune System

2.5 Antigen-Presenting Cells: Psoriatic Skin 24

2.5.1 Epidermal Dendritic Cells 24

2.5.2 Dermal Dendritic Cells (DDC) 28

2.6 Endothelial Cells $\quad 29$

2.7 Neutrophils $\quad 30$

2.8 Mast Cells 31

2.9 Keratinocytes 31

Chapter 3 Immune Cell Function in Psoriasis 34

3.1 T Lymphocytes: Peripheral Blood 34

3.2 T Lymphocytes: Skin $\quad 36$

3.2.1 T Cell Homing 36

3.2.2 T Cell Proliferation 40

3.2.3 Cytokine Production 45

3.3 Antigen-Presenting Cells 47

3.3.1 Epidermal Dendritic Cells 47

3.3.2 Dermal Dendritic Cells (DDC) 47

Chapter 4 Psoriasis is a T Cell-Mediated Disease 50

4.1 Anti-Psoriatic Treatments $\quad 50$

4.2 Anti-CD4 Monoclonal Antibodies 53

4.3 Lymphocyte-Selective Toxin (DAB $\left.{ }_{389} \mathrm{IL}-2\right)$

4.4 SCID Mouse Models $\quad 54$

4.5 Bone-Marrow Transplantation 56

4.6 T Cell Supernatants Promote Keratinocyte Proliferation $\quad 57$

4.7 Low $\mathrm{CD}^{+} / \mathrm{HIV}$ Infection $\quad 58$

Chapter 5 B Haemolytic Streptococci and Psoriasis 61

5.1 Group A Streptococci as a Trigger for

Guttate Psoriasis 61

5.2 Group A Streptococci and CP Psoriasis 63

5.3 Induction of Psoriatic Lesions by

Streptococci 
Contents ix

5.4 Streptococcal Antigens 64

5.4.1 Extracellular Products 64

5.4.1.1 Streptococcal pyrogenic

exotoxins (SPEs)

64

5.4.1.2 Streptococcal cytolytic toxins (haemolysins)

67

5.4.1.3 Streptococcal enzymes $\quad 68$

5.4.2 Cellular Proteins

69

5.4.2.1 $\mathrm{M}$ and $\mathrm{M}$-like proteins $\quad 70$

5.4.2.2 Other cell wall proteins $\quad 71$

5.4.2.3 Membrane proteins $\quad 72$

5.5 Immune Response to Extracellular

Streptococcal Proteins and Superantigens $\quad 73$

5.5.1 Humoral Response 73

5.5.2 In vivo/In vitro Response to SK/SD $\quad 74$

5.5.3 Superantigens

74

5.6 Immune Response to Streptococcal

Cellular Proteins

76

5.6.1 Streptococcal Cell Walls and

Membranes

76

5.6.2 Streptococcal M Protein 81

5.6.3 Streptococcal Membrane Proteins 85

5.6.4 CLA Induction by Group A

Streptococcal Antigens

86

Chapter 6 Immune Function of Psoriatic Keratinocytes 90

6.1 Cytokine Production 90

6.1.1 Interleukin-1 (IL-1) 92

6.1.2 Interleukin-6 (IL-6) 94

6.1.3 Interleukin-7 (IL-7) 95

6.1.4 Interleukin-8 (IL-8) 95

6.1.5 Interleukin-10 (IL-10) 97

6.1.6 Interleukin-12 (IL-12) 97

6.1.7 Interleukin-15 (IL-15) and
Interleukin-18 (IL-18)

6.1.8 Tumor Necrosis Factor- $\alpha$ (TNF- $\alpha$ ) 99 
$x$ Recent Advances in Psoriasis: The Role of the Immune System

6.1.9 Growth Factors

100

6.1.9.1 Transforming growth factor- $\alpha$ (TGF- $\alpha$ )/Epidermal growth factor (EGF)

6.1.9.2 Amphiregulin (AR)/ Heparin-binding EGF-like growth factor (HB-EGF)

101

6.1.9.3 Keratinocyte growth factor (KGF)

101

6.1.9.4 Insulin-like growth factor-I (IGF-I) receptor

102

6.1.9.5 Transforming growth factor- $\beta$ (TGF- $\beta$ )

102

6.1.10 Monocyte Chemotactic Protein-1 (MCP-1)/Monocyte Chemoattractant and Activating Factor (MCAF)

6.2 Response to Cytokines

6.2.1 Increased Proliferation

6.2.2 Decreased Proliferation

6.2.3 Surface Molecule Expression 107

6.2.4 Cytokine Production 108

6.3 Antigen/Superantigen Presentation 109

6.4 Integrins

6.5 Apoptosis

Chapter 7 Current and Future Immunological

Approaches to Treatment of Psoriasis

7.1 Current Treatments

7.1.1 PUVA and UVB

7.1.2 CyA and FK506

121

7.1.3 Steroid Hormones 124

7.1.3.1 Vitamin D analogues $\quad 124$

7.1.3.2 Vitamin A analogues $\quad 127$

7.1.3.3 Corticosteroids 128

7.1.4 Methotrexate

7.1.5 Dithranol 
Contents $x i$

7.2 Future Treatments 130

7.2.1 Cell Surface Molecule-Directed 130

7.2.1.1 CD4 130

7.2.1.2 Costimulatory molecules $\quad 132$

7.2.1.3 TCR 134

7.2.1.4 MHC Class II 135

7.2.1.5 Homing receptors 137

7.2.2 Cytokine-Directed 137

$\begin{array}{ll}\text { 7.2.2.1 IFN- } \gamma & 137\end{array}$

$\begin{array}{ll}\text { 7.2.2.2 TNF- } \alpha & 138\end{array}$

$\begin{array}{ll}7.2 .2 .3 \mathrm{IL}-10 & 138\end{array}$

7.2.2.4 IL-4 139

7.2.2.5 Toxin-conjugated cytokines 139

7.2.2.6 Conversion of $\mathrm{TH}_{1}$ to $\mathrm{TH}_{2}$ cytokine profile

7.2.3 Induction of Regulatory T Cells 141

7.2.3.1 T cell vaccination $\quad 141$

7.2.3.2 TCR peptides $\quad 142$

7.2.3.3 DNA encoding TCR V $\beta \quad 143$

7.2.3.4 Oral tolerance $\quad 143$

7.2.4 Apoptosis-Based Therapy 144

7.2.5 Gene Therapy 144

7.2.6 Purine Nucleoside Phosphorylase

Inhibitors/Ascomycins $\quad 145$

Chapter 8 Model for the Immunopathogenesis of Psoriasis 150

8.1 Outline of Proposed Model 150

8.2 Antigen Trigger: Group A Streptococci (GAS) 152

8.2.1 Streptococcal Superantigens Induce Psoriasis 153

8.2.2 Antigen-Specific Response 154

8.2.3 Perpetuation of the Psoriatic Process 155

8.2.3.1 Pathway $1 \quad 156$

8.2.3.2 Pathway $2 \quad 157$

8.2.3.3 Pathway $3 \quad 159$ 
xii Recent Advances in Psoriasis: The Role of the Immune System

8.3 $\mathrm{CD}^{+} \mathrm{T}$ Lymphocyte/KC Interaction

8.3.1 Surface Molecules

8.3.2 Cytokines

8.4 Role of $\mathrm{CD}^{+} \mathrm{T}$ Lymphocytes

8.5 Site of Altered Gene Expression

8.6 Other Antigenic Triggers

Index 